\title{
RADIO ISOTOPIC STUDY OF KIDNEY FUNCTION IN OBSTRUCTIVE JAUNDICE
}

\author{
By \\ El-Zawahry, M. D.; El Gendy, M.; Farid, M.; \\ Horia, H.; Abo El Hoda M.; El Laithy, R.; and El Diasty T.* \\ From \\ Department of General Surgery, Mansoura University \\ Hospital, and Radiology Department in Urology and Nephrology Unit, \\ Mansoura University
}

\section{INTRODUCTION}

Acute renal failure after biliary tract surgery was first described by Clairmont and VonHaberer (1911). The cause of supression of renal function in patients with obstructive jaundice is controversial. Dawson (1964) suggested defficient renal perfusion. He reported significant improvement of kindey function following mannitol diuresis. Endotoxaemia has a deleterious effect on renal function and structure. Endotoxaemia is due to absence of bile salts from the small itestine (Cavanough et al. 1970) and Mckay et al. (1966).

After biliary drainage (PTD), Lygidakis (1987) showed improvement of blood urea, serum creatinine and creatinine clearance. Thompson et al., (1986) using isotopic renal scan $99 \mathrm{mTC}$ DTPA showed that in jaun- diced patients G. F. R. was lower before biliary drainage than controls and that it increased significantly after drainage.

In this paper we studied renal function in 15 patients with obstructive jaundice before and after biliary drainage using isotopic renal scan in one surgical unit in Mansoura teaching hospital.

\section{Material and Methods}

This study was performed on 15 patients with obstructive jaundice and 12 non jaundiced renal transplant donors who acted as controls in Mansoura teaching hospital during the year 1989. Renal isotop scaning was done preoperatively and eight weeks after biliary drainage. 
t46!1 ә4t 4109 10$\rfloor$ 'saseo j01!

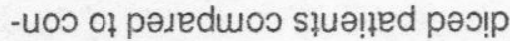
-une! st u! sełnuịu u! uo!̣e!nu

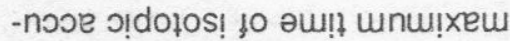

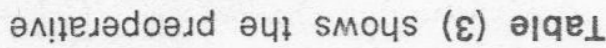

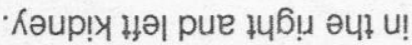

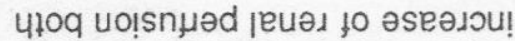

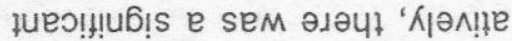

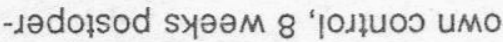
s!y se łua!ıed aut bu!ye $\perp$ 'sanjen

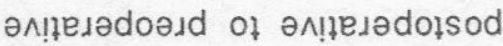
әчเ 6u!sedwos 'słuә!ฺе рәэ!р -une! st u! (o!̣ed eนoe/אәup!r)

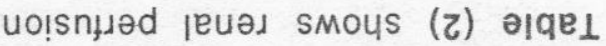

słua!ıed pəo!pune! u! uo!snuəd [euəd to uo!łonpad ұueo!!! łeul umous s! ł! 'sjoגłuos ţo łey!

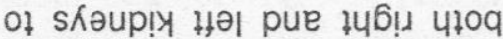
to o!ted uo!snuad әut bu!neduos -uọsnuəd ןеuәd pәseәләәр рец

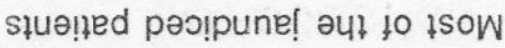
saseo joג słuə!ıed pos!̣une! st u! (o!ł -ed ejoe/Kaup!ry) uo!snjuad jeu

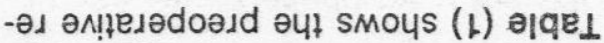

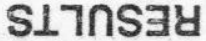

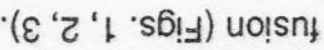

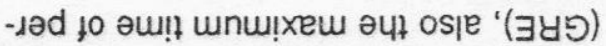

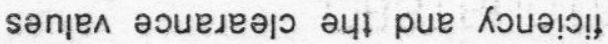

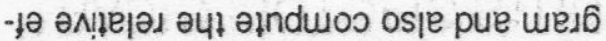

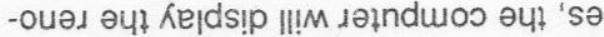

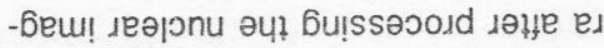

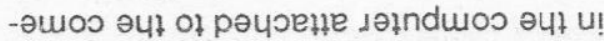

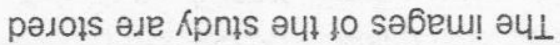

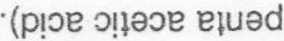

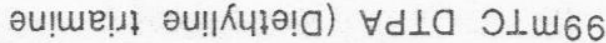
s! pesn әdolos!

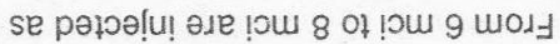

'uO!!

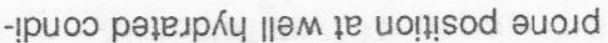

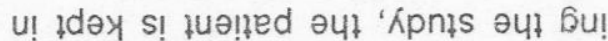

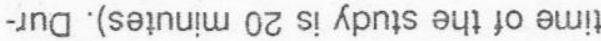

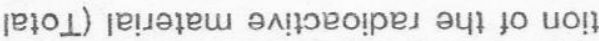
-eu!m!ן pue uo!nejnunose әцł słues

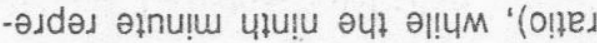
elose ol Кәup!Y) sরəup!y әut 10 әseyd uolsnuəd ә4! sMous Kpn!s au! to ə!n

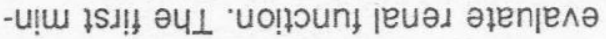

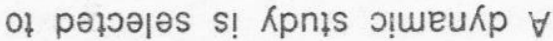

'słuejdsuedt ןeuəd to sıouop 乙! әдәм Kpnłs ग!dol

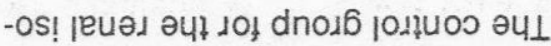

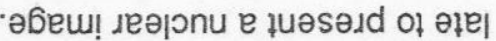
-nuоose I!!M słop y6nour әш!! pәu!m

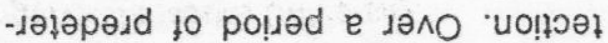

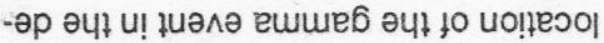

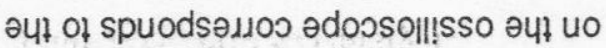

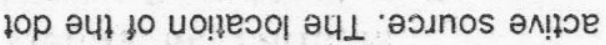

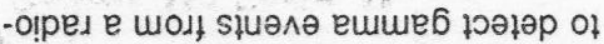

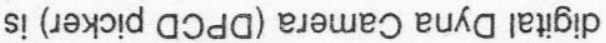

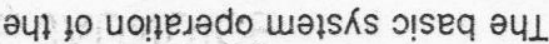

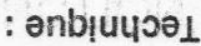


and left kidney there was a significant prolongation in maximum time of accumulation which reflects a diminished renal perfusion.

Table (4) shows the maximum time of isotopic accumulation in 15 jaundiced patients, comparing the postoperative to preoperative values. Taking the patient as his own control 8 weeks postoperatively, there was a significant shortening of maximum time of isotopic accumulation both in the right and left kindey indicating improvement in renal perfusion.

Table (5) Shows the preoperative isotopic clearance in 15 patients compared to control cases. It is shown that clearance value for the right kidney, the left kidney and the total clearance are decreased significantly when compared to controls.

Table (6) Shows isotopic clearance in 15 jaundiced patients, comparing the postoperative to preoperative values. Taking the patient as his own control, it is shown that clearance value for right and left kidney and total clearance are significantly in- creased postoperatively.

\section{DISCUSSION}

Altered renal perfusion has been suggested as a cause of hepatorenal syndrome in patients with obstructive jaundice. An increased sensitivitiy of vascular smooth muscle fibers to norepinephrine was found in rats when common bile duct was ligated (Gali et al. 1981).

A circulating factor could account for the changes in vascular reactivity. Bile salts may sensitize the kidney to ischaemic changes (Bomzon and kew, 1983; Aoyagi and Lowenstein, 1968). Other suggested factors included increased Renin level which produces afferent renal vasoconstriction (Berkowitz et al., 1974) vasoactive intestinal polypeptide (Hunt et al., 1979) kinin defficiency which produces decreased vasodilator activity (Wong et al., 1979).

In the present study, by performing renal isotopic scanning using $99 \mathrm{TCm}$ DTPA and estimating the renal perfusion as kidney/aorta ratio in 15 jaundiced patients, preoperatively there was a significant reduction of renal perfusion as compared to control group. Eight weeks after biliary drain- 


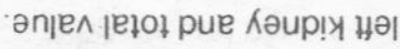

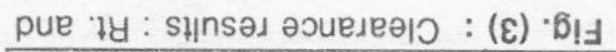

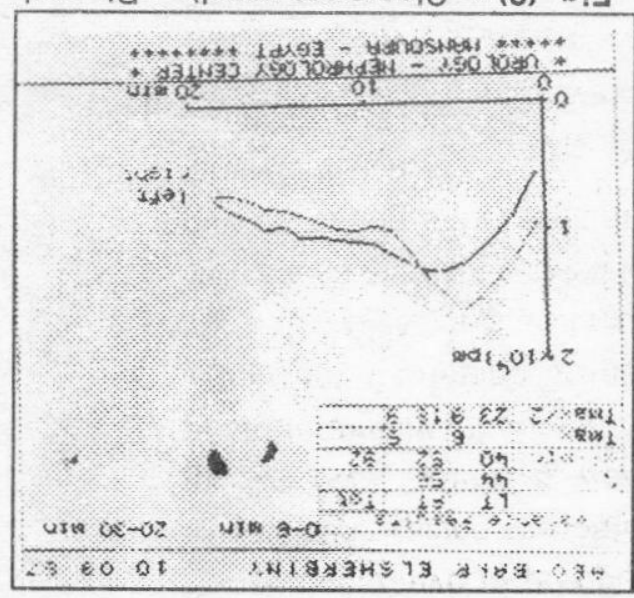

s|oגıoo se pәюе

pue sıouop łuejdsueג ןеuәд әдәм очм

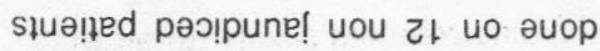
sem кpnıs әиł uo!!!ppe u әбeu!eıp

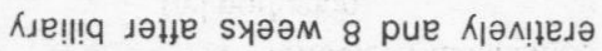
-doәдd әuop sem 6u!uueos ग!dolos! jeu

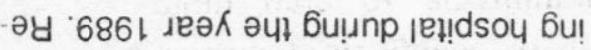
- чวеәц eınosupw u! pәџеәц әэ!pune!

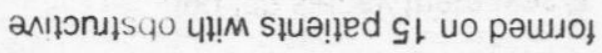
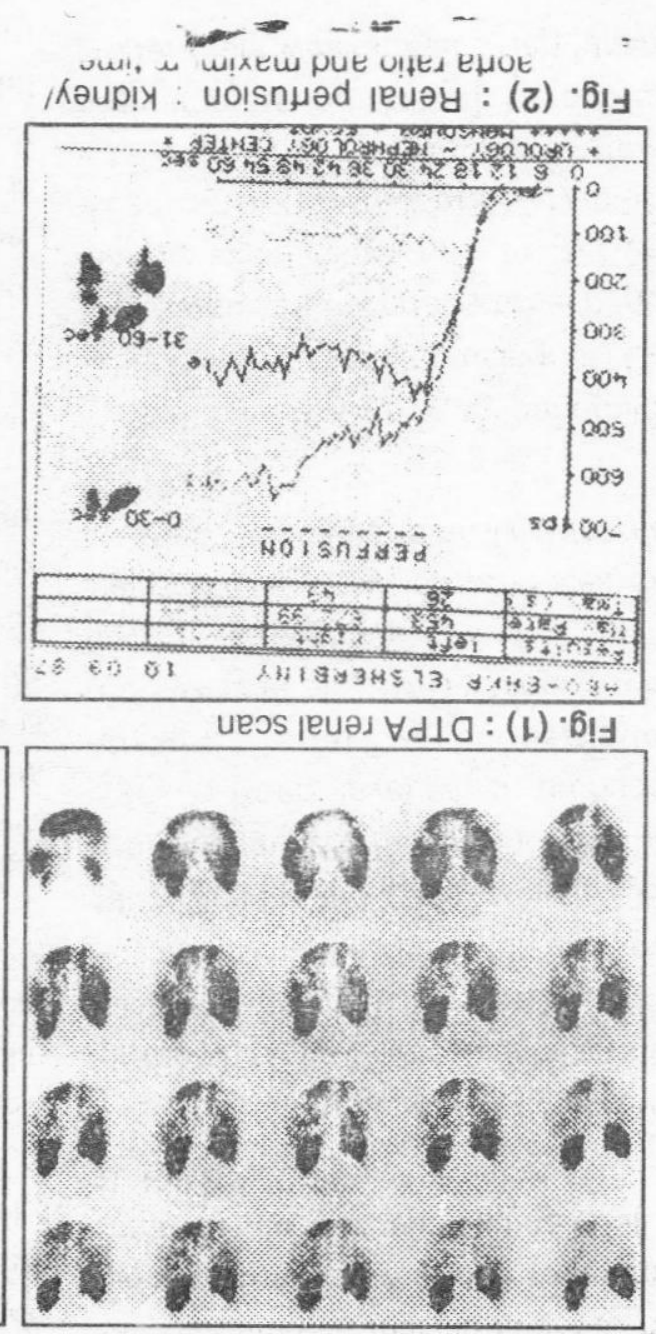

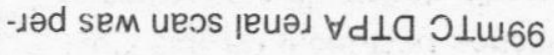

\section{Numuns}

uo!̣oun! Kaup!y pue uo!̣snyəd ןeu

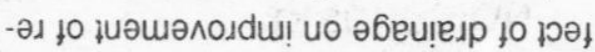

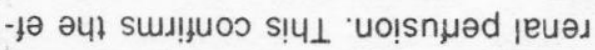

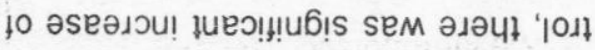
- иоэ uмо s! 
El-Zawahry, M. D et al....

Table (1) : Renel perhision (kidneylaorts retio) in 15 jeundiced patients Comparieon of preoperabve and control vaiues (12 cases)

\begin{tabular}{|c|c|c|c|c|}
\hline \multirow{2}{*}{$\begin{array}{l}\text { Case } \\
\text { No. }\end{array}$} & \multicolumn{2}{|c|}{ Aught kidhey } & \multicolumn{2}{|c|}{ Lett kodney } \\
\hline & Control & Pabent & Control & Patuent \\
\hline 1 & 3.2 & 26 & 3 & 1.8 \\
\hline 2 & 2.8 & 36 & 3.1 & 3.8 \\
\hline 3 & 31 & 2 & 3 & 1.5 \\
\hline 4 & 3.2 & 4 & 3 & 3 \\
\hline 5 & 2.8 & 2.8 & 28 & 3 \\
\hline 6 & 2.8 & 2.5 & 26 & 2 \\
\hline 7 & 3 & 2 & 3.2 & 2.1 \\
\hline B & 3 & 2.6 & 3.1 & 3.1 \\
\hline $\boldsymbol{\bullet}$ & 3.7 & 1.6 & 3.5 & 2.8 \\
\hline 10 & 3.2 & 2.5 & 2.9 & 1.6 \\
\hline 15 & 3.3 & 1.8 & 28 & 2 \\
\hline 12 & 4 & 2.1 & 2.9 & 1.5 \\
\hline 13 & & 2.25 & & 21 \\
\hline 14 & & 1.8 & & 2 \\
\hline 15 & & 2.5 & & 3 \\
\hline Mean & 3.183 & 2.45 & 2.992 & 2.32 \\
\hline $50 . \pm$ & 0.356 & 0.65 & 0.227 & 0.696 \\
\hline$P$ & & & & \\
\hline
\end{tabular}

Table (2) : Renal perfusion (kidney/aorta ratio) in 15 jaundiced patients comparison of Dreoperative and postoperative values

\begin{tabular}{|c|c|c|c|c|}
\hline \multirow{2}{*}{$\begin{array}{l}\text { Case } \\
\text { No. }\end{array}$} & \multicolumn{2}{|c|}{ Ploht kidney } & \multicolumn{2}{|c|}{ Latt kidney } \\
\hline & Proop. & Postop. & Preop. & Postop. \\
\hline 1 & 2.6 & 3 & 1.8 & 2.7 \\
\hline 2 & 3.6 & 3.8 & 3.8 & 3.8 \\
\hline 3 & 2 & 2 & 1.5 & 3.1 \\
\hline 4 & 4 & 3.8 & 3 & 3.4 \\
\hline 5 & 2.8 & 3 & 3 & 3.4 \\
\hline 6 & 2.5 & - & 2 & $\cdot$ \\
\hline 7 & 2 & $\cdot$ & 2.1 & $\cdot$ \\
\hline 8 & 2.6 & 3.5 & 3.1 & 3.2 \\
\hline 9 & 1.6 & 3.4 & 2.3 & 3.1 \\
\hline 10 & 2.5 & - & 1.5 & $\cdot$ \\
\hline 11 & 1.0 & 3.8 & 2 & 2.9 \\
\hline 12 & 2.1 & 2.5 & 1.5 & 2.4 \\
\hline 13 & 2.25 & 2.5 & 2.1 & 2.2 \\
\hline 14 & 1.8 & 2.8 & 2 & 3.1 \\
\hline 16 & 2.5 & 3 & 3 & 3.5 \\
\hline Mean & 2.45 & 3.04 & 2.32 & 2.95 \\
\hline S. D. \pm & 0.65 & c.zez & 0.696 & 0.403 \\
\hline$P$ & \multicolumn{2}{|c|}{$<0.05$} & $<0.05$ & \\
\hline
\end{tabular}


066 ' 1008 '

\begin{tabular}{|c|c|c|c|c|}
\hline \multicolumn{2}{|c|}{$\begin{array}{l}6 ! 5 \\
90^{\circ} 0>\end{array}$} & \multicolumn{2}{|c|}{$\begin{array}{c}6 ! 5 \\
50^{\circ} 0>\end{array}$} & \multirow[b]{2}{*}{$\begin{array}{l}\text { F.a. } \\
\text { ureew }\end{array}$} \\
\hline \multirow[t]{2}{*}{801.9} & $\begin{array}{l}89 \varepsilon^{\circ} \angle \\
\varepsilon \triangleright 9^{\circ} 8\end{array}$ & $\begin{array}{r}19 L^{\prime} \circ \\
99^{\circ} 8\end{array}$ & $\begin{array}{r}280^{\circ} L \\
\angle E 9{ }^{\circ} \mathrm{OL}\end{array}$ & \\
\hline & $L$ & s.9 & $\mathrm{Or}$ & St \\
\hline S:9 & 8 & $2 t$ & 81 & $\nabla 1$ \\
\hline 9 & OL & 8 & $\varepsilon\llcorner$ & $\varepsilon \downarrow$ \\
\hline $\mathrm{OL}$ & 22 & 8 & 22 & $2 t$ \\
\hline OL & 9 & 9 & $<$ & $1+$ \\
\hline$s$ & 62 & - & 62 & OL \\
\hline - & $L$ & 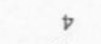 & 9 & 6 \\
\hline $\mathrm{g}$ & 9 & $\nabla t$ & 9 & 8 \\
\hline$b$ & 1 & - & t & $\angle$ \\
\hline - & OL & - & $\varepsilon^{\prime} \varepsilon \downarrow$ & 9 \\
\hline - & $99 \varepsilon$ & $s$ & 99 & g \\
\hline 8 & $\varepsilon \varepsilon^{\prime} L$ & 9.81 & 8 & $\triangleright$ \\
\hline s & $\varepsilon \varepsilon \varepsilon$ & s & $\varepsilon \varepsilon^{\prime} \varepsilon$ & $\varepsilon$ \\
\hline 87 & s & $\rightarrow$ & $99 \cdot 9$ & 2 \\
\hline$D$ & $\varepsilon \varepsilon \cdot \varsigma$ & $\angle$ & 901 & 1 \\
\hline dopeod & doodd & doysod & doosd & \\
\hline \multicolumn{2}{|c|}{ Көบря มөา } & \multicolumn{2}{|c|}{ Kөupp J46! } & eseo \\
\hline
\end{tabular}

-senfen enp̣ejedolsod pure enp̧esedoesd jo vosureduoo słuented

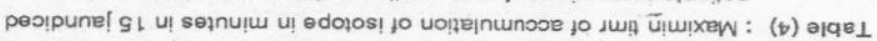

\begin{tabular}{|c|c|c|c|c|}
\hline \multicolumn{2}{|c|}{$\begin{array}{c}\text { 6!S } \\
90^{\circ} 0>\end{array}$} & \multicolumn{2}{|c|}{$\begin{array}{c}6 ! 5 \\
50^{\circ} 0>\end{array}$} & $d$ \\
\hline $89 \varepsilon^{\circ} L$ & $820^{\circ} \mathrm{L}$ & $287^{\circ} \mathrm{L}$ & t9S' ! & F. \\
\hline $\operatorname{ct} 8$ & 690.9 & $\angle 89^{\circ} O \mathrm{~L}$ & ટદદ's & ueeW \\
\hline$\angle$ & & OL & & St \\
\hline 8 & & 81 & & $\nabla t$ \\
\hline OL & & $\varepsilon \vdash$ & & $\varepsilon_{l}$ \\
\hline 22 & s & 22 & $\triangleright$ & Zl \\
\hline 9 & 9 & $L$ & 8 & bL \\
\hline 62 & S & 62 & $\mathbf{S}$ & Or \\
\hline$L$ & $\mathbf{S}$ & 9 & s & 6 \\
\hline S & $\varepsilon \varepsilon^{*} L$ & 9 & 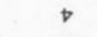 & 8 \\
\hline$\downarrow$ & $\mathrm{S}^{\prime} \varepsilon$ & b & s & $\angle$ \\
\hline Ol & $\forall$ & $\varepsilon^{\prime} \varepsilon\llcorner$ & 8 & 9 \\
\hline $99^{\circ} \varepsilon$ & 9 & 9 'S & $L$ & S \\
\hline$\varepsilon \varepsilon^{*} L$ & S & s & 995 & $\triangleright$ \\
\hline$\varepsilon \varepsilon^{\prime} \varepsilon$ & $\nabla$ & $\varepsilon \varepsilon^{\prime} \varepsilon$ & $\varepsilon \varepsilon^{*} \varepsilon$ & $\varepsilon$ \\
\hline S & S & $99: 9$ & $\nabla$ & 2 \\
\hline$\varepsilon \varepsilon^{\prime} s$ & s & 901 & s & $\downarrow$ \\
\hline squeped & 10nuos & jueped & 104uos & ${ }^{\circ} \mathrm{ON}$ \\
\hline \multicolumn{2}{|c|}{ Көuppy yөา } & \multicolumn{2}{|c|}{ Kөupp גบচi! } & eseo \\
\hline
\end{tabular}

- (seses

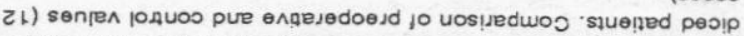

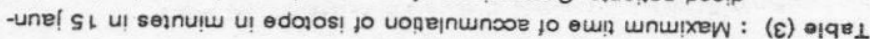


Table (5) : Isotopic clearance value in 15 jaundiced patients comparison between preoperative and control values (12 cases).

\begin{tabular}{|c|c|c|c|c|c|c|}
\hline \multirow{2}{*}{$\begin{array}{c}\text { Case } \\
\text { No. }\end{array}$} & \multicolumn{2}{|c|}{ Right kidney } & \multicolumn{2}{|c|}{ Left kidney } & \multicolumn{2}{|c|}{ Total clearance } \\
\hline & Control & patient & Control & patient & Control & patient \\
\hline 1 & 56 & 60 & 50 & 32 & 106 & 92 \\
\hline 2 & 54 & 56 & 51 & 49 & 105 & 105 \\
\hline 3 & 68 & 49 & 63 & 20 & 131 & 69 \\
\hline 4 & 63 & 44 & 48 & 37 & 111 & 81 \\
\hline 5 & 44 & 75 & 35 & 28 & 79 & 103 \\
\hline 6 & 47 & 46 & 30 & 25 & 77 & 71 \\
\hline 7 & 57 & 10 & 61 & 12 & 118 & 22 \\
\hline 8 & 46 & 32 & 58 & 40 & 104 & 72 \\
\hline 9 & 71 & 43 & 62 & 36 & 133 & 79 \\
\hline 10 & 64 & 49 & 43 & 29 & 107 & 78 \\
\hline 11 & 60 & 38 & 40 & 34 & 100 & 72 \\
\hline 12 & 54 & 16 & 40 & 14 & 94 & 30 \\
\hline 13 & & 30 & & 23 & & 53 \\
\hline 14 & & 32 & & 33 & & 65 \\
\hline 15 & . & 27 & & 27 & & 54 \\
\hline Mean & 57 & 40.47 & 48.42 & 29.27 & 105.33 & 69.73 \\
\hline S. D. \pm & 8.63 & 16.86 & 11.07 & 9.77 & 17.26 & 23.22 \\
\hline$P$ & \multicolumn{2}{|c|}{$\begin{array}{c}<0.05 \\
\text { Sig. }\end{array}$} & \multicolumn{2}{|c|}{$<0.05$} & \multicolumn{2}{|c|}{$\begin{array}{c}<0.05 \\
\text { Sig. }\end{array}$} \\
\hline
\end{tabular}

Table (6) : Isotopic clearance value in 15 jaundiced patients comparison between postoperative and preoperative values.

\begin{tabular}{|c|c|c|c|c|c|c|}
\hline \multirow{2}{*}{$\begin{array}{c}\text { Case } \\
\text { No. }\end{array}$} & \multicolumn{2}{|c|}{ Right kidney } & \multicolumn{2}{|c|}{ Left kidney } & \multicolumn{2}{|c|}{ Total clearance } \\
\hline & Preop. & Postop. & Proop. & Postop. & Preop. & Postop. \\
\hline 1 & 60 & 60 & 32 & 45 & 92 & 105 \\
\hline 2 & 56 & 60 & 49 & 50 & 105 & 110 \\
\hline 3 & 49 & 52 & 20 & 38 & 69 & 90 \\
\hline 4 & 44 & 43 & 37 & 26 & 81 & 69 \\
\hline 5 & 75 & 80 & 28 & 58 & 103 & 138 \\
\hline 6 & 46 & $\cdot$ & 25 & $\cdot$ & 71 & $\cdot$ \\
\hline 7 & 10 & $\cdot$ & 12 & - & 22 & $\cdot$ \\
\hline 8 & 32 & 56 & 40 & 34 & 72 & 90 \\
\hline 9 & 43 & 66 & 36 & 52 & 79 & 118 \\
\hline 10 & 49 & $\cdot$ & 29 & - & 78 & - \\
\hline 11 & 38 & 43 & 34 & 44 & 72 & 87 \\
\hline 12 & 16 & 36 & 14 & 48 & 30 & 84 \\
\hline 13 & 30 & 32 & 23 & 27 & 53 & 59 \\
\hline 14 & 32 & 50 & 33 & 56 & 65 & 106 \\
\hline 15 & 27 & 35 & 27 & 40 & 54 & 75 \\
\hline Mean & 40.47 & 51.8 & 29.27 & 42.8 & 69.73 & 91.6 \\
\hline S. D. \pm & 16.86 & 14.46 & 9.77 & 11.33 & 23.22 & 18.29 \\
\hline$P$ & \multicolumn{2}{|c|}{$\begin{array}{c}<0.05 \\
\text { Sig. }\end{array}$} & \multicolumn{2}{|c|}{$\begin{array}{c}<0.05 \\
\text { Sig. }\end{array}$} & \multicolumn{2}{|c|}{$\begin{array}{c}<0.05 \\
\text { Sig. }\end{array}$} \\
\hline
\end{tabular}




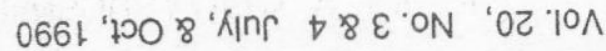

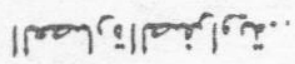

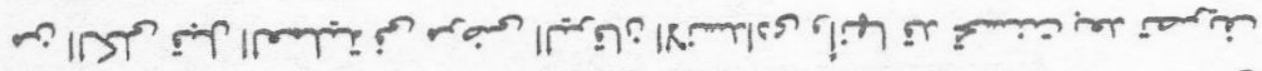

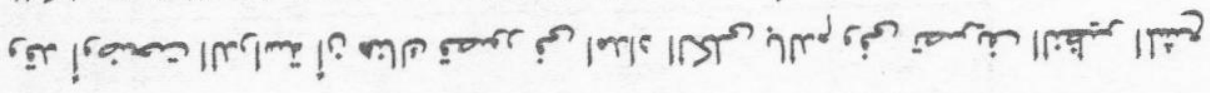

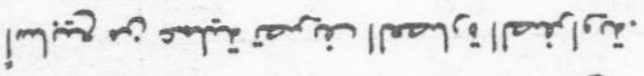

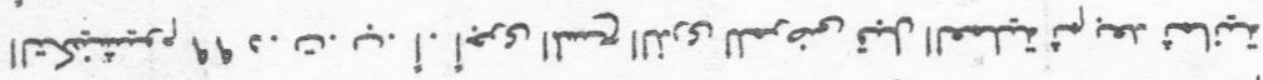
רז |

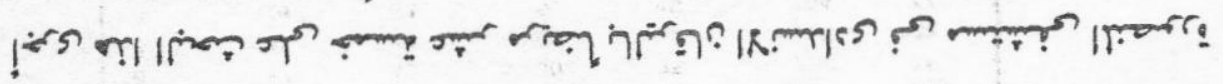

rराm

Imañ 